\section{Consumo alimentar de crianças \\ e adolescentes residentes em \\ uma área de invasão em Maceió, \\ Alagoas, Brasil}

\author{
Food consumption of children and \\ adolescents living in an area of \\ invasion in Maceio, Alagoas, Brazil
}

Juliana Vasconcelos Lyra da Silva'

Anny Karinny Calheiros Dutra Timóteo"

Célia Dias dos Santos"

Gilberto Fontes'

\section{Eliana Maria Maurício da Rocha'}

'Instituto de Ciências Biológicas e da Saúde da Universidade Federal de Alagoas. Maceió, Alagoas, Brasil.

"Faculdade de Nutrição da Universidade Federal de Alagoas. Maceió, Alagoas, Brasil.

\section{Resumo}

Objetivo: Avaliar o consumo alimentar de energia, macronutrientes e micronutrientes, relacionados ao crescimento e desenvolvimento, de indivíduos menores de 16 anos residentes numa área de invasão consolidada em Maceió, Alagoas. Métodos: Estudo do tipo transversal, realizado com 272 indivíduos menores de 16 anos residentes numa área de invasão consolidada em Maceió, Alagoas. A avaliação do consumo alimentar foi realizada pelo método recordatório 24 horas, com auxílio de um álbum de registro fotográfico. Para a análise da composição nutricional das dietas utilizou-se o programa Virtual-Nutri 1.0. A estimativa da prevalência de inadequação foi baseada nas Ingestões Dietéticas de Referência (DRIs) com o ajuste da variabilidade intrapessoal. Para a análise dos dados utilizou-se o programa Statistical Package for Social Science (SPSS) versão 11.5. Resultados: Dos indivíduos avaliados, $5,6 \%$ apresentavam ingestão energética excessiva e 3,7\% insuficiente. A frequência de ingestão energética excessiva foi superior no grupo de 1 a 3 anos $(p<0,05)$, que também apresentou maiores frequências de ingestão inadequada para vitamina $\mathrm{A}$, vitamina $\mathrm{E}$ e zinco, e foi a única faixa etária que teve consumo médio de cálcio superior à ingestão adequada. Conclusão: os dados demonstram que o padrão alimentar dos indivíduos avaliados necessita de ajustes.

Palavras-chave: Ingestão alimentar. Consumo energético. Macronutrientes. Micronutrientes. 


\section{Abstract}

Objective: To assess the food intake of energy, macronutrients and micronutrients related to growth and development in individuals under 16 years of age living in a consolidated invasion settlement in Maceió, Alagoas. Methods: The assessment of food intake was carried out by the 24-hour recall method, using a food picture album. Nutritional composition of diets was analysed using the Virtual Nutri-1.0 software. The estimated prevalence of inadequate intake was based on the Dietary Reference (DRIs), adjusted to account for intra-individual variability. The Statistical Package for Social Science (SPSS) version 11.5 was used for data analysis. Results: $5.6 \%$ of the subjects studied presented excessive energy intake and for $3.7 \%$ it was inadequate. The frequency of excessive energy intake was higher in the 1 to 3 yearold age group ( $\mathrm{p}<0.05)$, which also showed higher rates of inadequate intake of vitamin A, vitamin E and zinc, and it was the only group in which average consumption of calcium was higher than the adequate intake. Conclusion: Data show that dietary patterns of the individuals studied need adjustments.

Keywords: Food intake. Energy. Macronutrients. Micronutrients.

\section{Introdução}

A avaliação do consumo alimentar pode ser utilizada como indicador indireto do estado nutricional, capaz de detectar situações de risco na alimentação de indivíduos e de coletividades. O conhecimento da ingestão de nutrientes permite que se estabeleça o diagnóstico nutricional com o objetivo de formular medidas capazes de promover as mudanças desejáveis no comportamento alimentar. Mais que isso, auxilia no planejamento e definições de políticas de saúde pública e de ações de intervenção ${ }^{1,2}$.

É importante a caracterização do estado nutricional durante a infância e a adolescência, pois uma alimentação balanceada em energia e nutrientes é essencial para o pleno crescimento e desenvolvimento dos indivíduos nestas fases da vida ${ }^{3}$. Tal avaliação pode sofrer interferência de fatores como diversidade da dieta, hábitos alimentares, qualidade da informação, idade, imagem corporal, memória do entrevistado, crenças, comportamento, cultura e nível socioeconômico, bem como fatores de exposição. Desta forma, o ato de registrar a ingestão alimentar de um indivíduo torna-se complexo ${ }^{4}$.

Para estimar a prevalência de inadequação da ingestão de um determinado nutriente, é necessário calcular seu consumo pelo grupo populacional de interesse e compará-lo com os padrões de referência. Entretanto, os indivíduos frequentemente variam o tipo e a quantidade de alimentos que consomem diariamente, ocorrendo grande variabilidade no consumo dos nutrientes pela mesma pessoa (intrapessoal) e entre as pessoas (interpessoal) ${ }^{5,6}$.

Hoffmann e cols. relatam que a repetição do recordatório de 24 horas (REC24h) pode ser utilizada para descrever a ingestão dietética habitual e recomendam a utilização de dois REC24h por indivíduo, em dias não consecutivos, para estimar o consumo em todos os dias da semana ${ }^{7}$.

Recentemente estudos foram realizados no intuito de caracterizar o consumo alimentar de crianças e adolescentes, demonstrando que hábitos alimentares inade- 
quados estão entre os fatores determinantes do estado nutricional ${ }^{2,8,9}$. Entretanto, tais estudos não consideraram a variabilidade intrapessoal.

O presente estudo se propõe a avaliar o consumo alimentar de energia, macronutrientes e micronutrientes, relacionados ao crescimento e desenvolvimento, em crianças e adolescentes, residentes numa área de invasão consolidada em Maceió, Alagoas, levando em consideração a variabilidade intrapessoal.

\section{Métodos}

Trata-se de um estudo transversal realizado em 2005, envolvendo 272 indivíduos menores de 16 anos, isto é, crianças, pré-adolescentes e adolescentes na fase inicial, residentes em uma área de invasão consolidada da cidade de Maceió, Estado de Alagoas. A área estudada pertence à região denominada Vale do Reginaldo, às margens de um canal localizado em parte do bairro do Feitosa e do Farol. Esta área é caracterizada por encostas e uma parte plana, com ocupação irregular e habitações espalhadas de maneira desordenada, semelhante à situação de assentamentos subnormais. $\mathrm{O}$ canal foi construído para conduzir água pluvial precipitada em sua bacia; entretanto, é utilizado para escoamento de bairros da cidade que não possuem saneamento básico.

Selecionou-se uma subárea de aproximadamente $60.000 \mathrm{~m}^{2}$, e do total de 367 indivíduos menores de 16 anos cadastrados, 272 participaram do estudo.

Os pesquisadores envolvidos na coleta dos dados participaram de treinamentos teórico-práticos e de uma pesquisa piloto visando a uniformização da conduta. Um manual de coleta de dados foi elaborado exclusivamente para o estudo.

A avaliação do consumo alimentar foi realizada pelo método REC24h ${ }^{10}$. Os dados foram coletados por meio de entrevistas domiciliares, com utilização de um formulário no qual eram anotados os alimentos consumidos, formas de preparo, quantidades consumidas e marcas comerciais dos alimentos industrializados. Para os menores de 10 anos, a entrevista foi realizada com a mãe ou o responsável, na presença da criança. Foram excluídos do estudo dez indivíduos menores de um ano devido à ausência de EAR para esta faixa etária.

Para a padronização das porções dos alimentos utilizou-se o Álbum de Registro Fotográfico ${ }^{11}$, com a inclusão dos alimentos regionais reconhecidos como de maior consumo por populações de baixa renda no Nordeste brasileiro. Alimentos tais como cuscuz, batata doce, inhame, charque e mortadela foram pesados em duplicata, fotografados e anexados ao Álbum. Para a pesagem dos alimentos utilizou-se uma balança digital (Marte Balanças e Aparelhos de Precisão Ltda.) com capacidade máxima de 5.000g e sensibilidade de $1 \mathrm{~g}$.

As medidas caseiras foram transformadas em porções, quantidade em grama (g) ou volume em mililitro $(\mathrm{mL})$, para subsequente análise de energia e nutrientes. Foram analisados: carboidratos (HC), proteínas (PTN), lipídios (LIP), vitamina A (vitA), vitamina E (vitE), ferro (Fe), zinco (Zn) e cálcio (Ca). O critério adotado para seleção destes nutrientes foi a relação com o crescimento e desenvolvimento das crianças e adolescentes. Foi analisada a ingestão de vitamina C (vitC) devido ao importante papel desta vitamina na absorção de Fe.

A análise da composição nutricional das dietas foi realizada pelo programa VirtualNutri $1.0^{12}$, sendo inseridos os dados de alimentos não encontrados no programa a partir de uma sequência predefinida de tabelas de composição química de alimen$\operatorname{tos}^{13-16}$. Foram utilizados os dados presentes nos rótulos dos alimentos industrializados, quando estes não constavam nas tabelas. No caso de preparações ausentes, tanto no programa quanto nas tabelas, a composição nutricional do alimento foi obtida através da receita. A composição energética foi identificada pelo consumo de calorias fornecidas por HC, PTN e LIP.

Para a análise de prevalência de inadequação da ingestão de nutrientes foram utilizadas as recomendações do Institute 
of Medicine (IOM), denominadas DRIs (Dietary Reference Intake) ${ }^{17}$. Os macronutrientes foram avaliados através da AMDR (Acceptable Macronutrient Distribution Range) e os nutrientes PTN, Fe, Zn, vitA, vitC e vitE pelo método EAR (Estimated Average Requirement), como ponto de corte conforme proposto por Beaton ${ }^{18}$. Devido à inexistência de EAR para o $\mathrm{Ca}$, não foi possível estimar a prevalência de inadequação para este mineral, portanto os valores médios de consumo foram comparados com a AI (Adequate Intake). Todos os dados de consumo alimentar foram corrigidos pela variabilidade intrapessoal.

Para avaliação de energia utilizou-se o marcador biológico, peso corporal, através do cálculo do índice de massa corporal $(\mathrm{IMC})^{19}$. Este marcador indica equilíbrio ou desequilíbrio entre ingestão e gasto de energia. Caracteriza-se a ingestão insuficiente quando o IMC está abaixo de -2DP, ingestão adequada se IMC fica entre -2DP e 2DP e ingestão excessiva quando IMC está acima de 2DP. Devido à perda de seguimento participaram desta avaliação 216 indivíduos dos 272 participantes do estudo.

Para cálculo do IMC, o peso das crianças menores de 24 meses foi aferido em balança digital infantil (Filizola $\left.{ }^{\circledR}\right)$, com capacidade máxima de $16 \mathrm{Kg}$ e sensibilidade de $10 \mathrm{~g}$, e o comprimento medido em posição de decúbito dorsal em estadiômetro pediátrico de madeira de $120 \mathrm{~cm}$, com escala milimétrica em fibra de vidro. Para participantes com idade igual ou maior que 24 meses, o peso corporal foi tomado em balança digital plataforma (Filizola ${ }^{\circledR}$ ), com capacidade máxima de $150 \mathrm{Kg}$ e sensibilidade de 100 g, e os valores da estatura obtidos em posição ortostática em estadiômetro vertical (WCS ${ }^{\circledR}$ ), com $212 \mathrm{~cm}$ de altura e escala em milímetro.

A análise dos dados foi realizada com auxílio do programa Statistical Package for Social Science (SPSS) versão $11.5^{20}$. Utilizouse o teste $\chi^{2}$ (Qui quadrado) de Pearson para comparação das frequências.

Os responsáveis pelas crianças e adolescentes foram informados e esclarecidos sobre o propósito do estudo, sendo o consentimento obtido através da assinatura do Termo de Consentimento Livre e Esclarecido. O presente estudo foi aprovado pelo Comitê de Ética em Pesquisa da Universidade Federal de Alagoas, em 8 de julho de 2004, registro ${ }^{\circ}$ 006491/2004-85.

\section{Resultados e Discussão}

As DRIs fornecem valores de referência, recomendados para prevenção de deficiências nutricionais e doenças crônicas não transmissíveis, além de estabelecer limites máximos para a ingestão de nutrientes, minimizando os riscos de efeitos adversos de toxicidade provinda da ingestão excessiva. Entretanto, estas referências foram estabelecidas para a população americana e canadense e sua utilização na população brasileira deve levar em consideração possíveis diferenças nos padrões alimentares ${ }^{17}$.

Dos 216 indivíduos avaliados quanto à ingestão energética, 196 (90,7\%) apresentavam ingestão adequada e 12 (5,6\%) ingestão excessiva (Figura 1). O consumo excessivo de energia, especialmente nos estágios mais precoces da vida, pode facilitar o sobrepeso e, desta forma, tornar-se um fator de risco para doenças, particularmente aquelas de origem metabólicas na vida adulta ${ }^{21}$. A utilização do recordatório de 24 horas tende a subestimar o consumo de energia, devido a lapsos de memória e dificuldade na quantificação dos alimentos consumidos; por isso, é possível que a frequência do consumo excessivo de energia seja ainda maior.

O consumo adequado ou excessivo de energia não garante a adequação de micronutrientes, podendo estar associado à ingestão de alimentos que fornecem "calorias vazias", isto é, ricos em energia e pobres em micronutrientes. Tal condição favorece o aparecimento da denominada "fome oculta", representada pela carência de micronutrientes na presença de adequação calórica.

A frequência encontrada de indivíduos com ingestão energética insuficiente foi de 3,7\% (Figura 1). Indivíduos nesta situação 


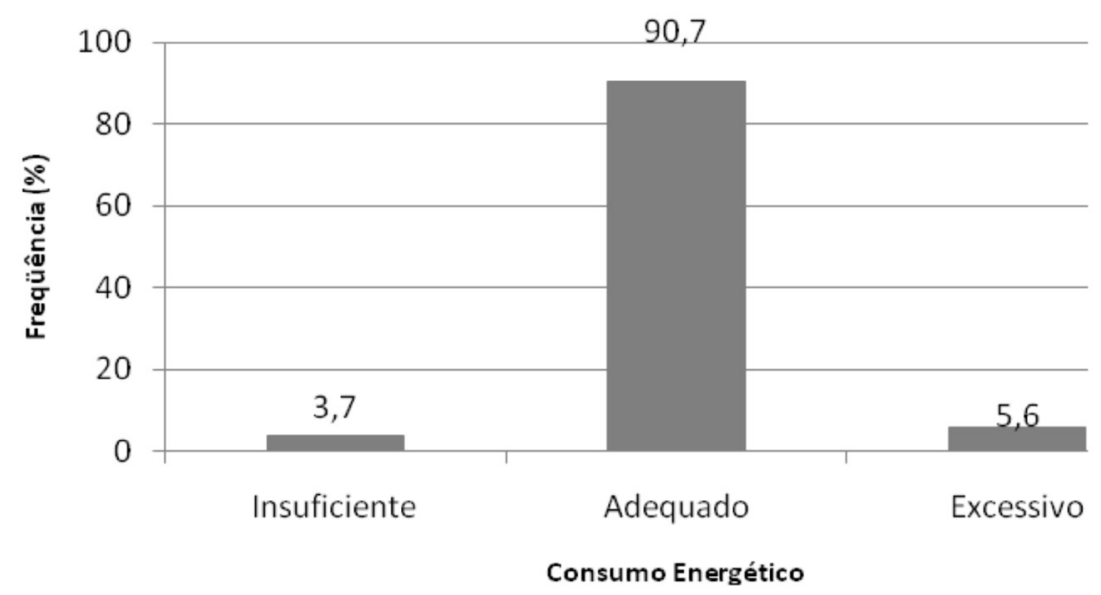

Figura 1 - Distribuição do consumo energético em indivíduos menores de 16 anos, residentes numa área de invasão em Maceió, Alagoas, 2005.

Figure 1 - Distribution of energy consumption in individuals under 16 years of age living in an area of invasion in Maceio, Alagoas, 2005.

apresentam risco de desenvolver desnutrição energético-protéica, o que afetaria o crescimento e desenvolvimento das crianças e adolescentes.

Levy-Costa e cols. registraram as mudanças no padrão alimentar da população brasileira, que incluem aumento no consumo de alimentos industrializados e diminuição na ingestão de frutas e hortaliças ${ }^{22}$. Informações disponíveis acerca do consumo alimentar de crianças e adolescentes, revelam ingestão excessiva de alimentos densamente energéticos, ricos em gorduras e açúcares simples ${ }^{23,24}$. Corroborando com estas informações, encontrou-se no presente estudo ingestão energética excessiva nos grupos de 1 a 3 anos e de 14 a 15 anos (Tabela 1).
A Tabela 2 apresenta a distribuição da participação de macronutrientes abaixo da AMDR, de acordo com as faixas etárias. Observa-se que $2,9 \%, 49,3 \%$ e $1,8 \%$ da população estudada (272) apresentavam, respectivamente, percentuais de HC, LIP e PTN provenientes do valor energético total abaixo da AMDR. Quando analisada por faixa etária, esta distribuição foi estatisticamente diferente apenas para os LIP $(\mathrm{p}<0,05)$, sendo mais frequente no grupo de 1 a 3 anos (64,3\%). Ingestão acima ou abaixo da AMDR aumenta o risco do aparecimento de doenças crônicas e pode resultar na ingestão inadequada de nutrientes essenciais ${ }^{19}$.

As Tabelas 3 e 4 apresentam a necessidade média estimada (EAR) e a média

Tabela 1 - Consumo energético médio e freqüência de ingestão energética inadequada, de acordo com faixas etárias, em indivíduos menores de 16 anos, residentes numa área de invasão em Maceió, Alagoas, 2005.

Table 1 - Average energy intake and frequency of inadequate intake according to age group in individuals under 16 years of age living in an area of invasion in Maceio, Alagoas, 2005.

\begin{tabular}{lcccccccc}
\hline \multirow{2}{*}{$\begin{array}{l}\text { Faixa Etária } \\
\text { (anos) }\end{array}$} & $\mathrm{n}$ & Média $\pm \mathrm{DP}($ Kcal/dia) & \multicolumn{6}{c}{ Consumo energético } \\
\cline { 4 - 8 } & & & $\mathrm{n}$ & $\%$ & $\mathrm{n}$ & $\%$ & $\mathrm{n}$ & $\%$ \\
\hline $1-13$ & 45 & $1.260,6 \pm 440,5$ & 0 & 0,0 & 38 & 84,4 & 7 & 15,6 \\
$3 \dashv 8$ & 84 & $1.470,4 \pm 219,9$ & 3 & 3,6 & 80 & 95,2 & 1 & 1,2 \\
$8 \dashv 13$ & 80 & $1.542,3 \pm 405,5$ & 4 & 5,0 & 73 & 91,3 & 3 & 3,8 \\
$13-15$ & 7 & $1.720,1 \pm 403,2$ & 1 & 14,3 & 5 & 71,4 & 1 & 14,3 \\
\hline${ }^{*} \mathrm{p}<0,05$ & & & & & & & &
\end{tabular}


Tabela 2 - Distribuição dos indivíduos menores de 16 anos, residentes numa área de invasão em Maceió, segundo a participação de macronutrientes abaixo da faixa de distribuição aceitável de macronutrientes (AMDR), de acordo com faixas etárias.

Table 2 - Distribution of individuals under 16 years of age living in an area of invasion in Maceio, according to the participation of nutrients below Acceptable Macronutrient Distribution Ranges (AMDR), according to age group.

\begin{tabular}{|c|c|c|c|c|c|c|}
\hline \multirow{3}{*}{$\begin{array}{l}\text { Faixa Etária } \\
\text { (anos) }\end{array}$} & \multicolumn{6}{|c|}{ Consumo abaixo da AMDR } \\
\hline & \multicolumn{2}{|c|}{ Carboidratos } & \multicolumn{2}{|c|}{ Lipídios* } & \multicolumn{2}{|c|}{ Proteínas } \\
\hline & $\mathrm{n}$ & $\%$ & $\mathrm{n}$ & $\%$ & $\mathrm{n}$ & $\%$ \\
\hline $1-3$ & 3 & 5,4 & 36 & 64,3 & 0 & 0,0 \\
\hline $3-8$ & 1 & 0,9 & 41 & 38,7 & 4 & 3,8 \\
\hline $8 \dashv 13$ & 3 & 3,0 & 53 & 53,5 & 1 & 1,0 \\
\hline $13-15$ & 1 & 9,1 & 4 & 36,4 & 0 & 0,0 \\
\hline TOTAL & 8 & 2,9 & 134 & 49,3 & 5 & 1,8 \\
\hline
\end{tabular}

de consumo de nutrientes, segundo faixa etária e sexo, nos 272 indivíduos avaliados. Já a Figura 2 apresenta a frequência de ingestão inadequada segundo faixa etária nos mesmos indivíduos.

Apesar do consumo médio de PTN ter sido superior à recomendação em todas as faixas etárias para ambos os sexos (Tabelas 3 e 4), a freqüência de ingestão protéica inadequada variou de $28,6 \%$ a $47,8 \%$ (Figura 2 ) entre as faixas etárias $(\mathrm{p}>0,05)$.

O consumo de PTN possui relação com a ingestão energética. Quando não há o consumo adequado de energia as PTN são utilizadas para suprir esta necessidade, prejudicando sua função na construção de novos tecidos. Dessa maneira, a relação síntese e deposição de PTN é diretamente influenciada pelo valor energético da alimentação ${ }^{25,26}$.

Entre as vitaminas estudadas, apenas a vitA, apresentou média de consumo superior à recomendação em todas as faixas etárias em ambos os sexos (Tabelas 3 e 4). A distribuição da ingestão inadequada de vitA foi diferente entre as faixas etárias $(\mathrm{p}<0,05)$, com frequência maior $(49,7 \%)$ nos indivíduos de 1 a 3 anos (Figura 2). Esta vitamina desempenha papel essencial na diferenciação celular normal, no crescimento e desenvolvimento ósseo, nos processos imunológicos, na visão e na reprodução ${ }^{27}$. Devido às suas funções, seu consumo deve ser adequado em qualquer fase da vida, principalmente naqueles que estão em crescimento. Existem evidências de que a deficiência de vitA altera o metabolismo normal do Fe, por mecanismos não totalmente esclarecidos, ocasionando um quadro de anemia ${ }^{28,29} \mathrm{e} o$ excesso na ingestão desta vitamina pode causar alterações hepáticas com efeitos $\operatorname{adversos}^{30}$.

A média de ingestão da vitE, foi inferior à recomendação em todas as faixas etárias para ambos os sexos (Tabelas 3 e 4). A frequência de ingestão inadequada de vitE foi superior a $25 \%$ em todos os grupos, sendo a diferença entre eles estatisticamente significativa $(\mathrm{p}<0,05)$, atingindo $100 \%$ dos indivíduos de 1 a 3 anos (Figura 2). Esta vitamina, além de possuir papel estrutural, protege a integridade de membranas celulares devido à sua ação antioxidante, desempenhando importante papel na imunocompetência. O comprometimento das defesas imunológicas facilita a instalação dos processos infecciosos que comprometem o estado nutricional $^{31,32}$.

Com relação ao consumo de vitC, a média de ingestão foi inferior à recomendação nos indivíduos de ambos os sexos do grupo etário de 9 a 13 e 14 a 15 anos (Tabelas 3 e 4). $\mathrm{O}$ consumo inadequado distribuiu-se diferentemente entre as faixas etárias $(\mathrm{p}<0,05)$, 
sendo mais frequente no grupo de 14 a 15 anos, afetando quase metade dos indivíduos (45,5\%) (Figura 2). A vitC ou ácido ascórbico, devido ao seu alto poder redutor, participa da absorção do Fe não heme e proporciona proteção contra oxidação descontrolada no meio aquoso da célula. $O$ consumo inadequado pode minimizar a absorção de Fe

Tabela 3 - Necessidade média estimada (EAR) e média de consumo de nutrientes, de acordo com faixas etárias, em indivíduos menores de 16 anos do sexo masculino, residentes numa área de invasão em Maceió, Alagoas, 2005.

Table 3 - Estimated Average Requirement (EAR) and average consumption of nutrients according to age group, in male individuals under 16 years of age living in an area of invasion in Maceio, Alagoas, 2005.

\begin{tabular}{|c|c|c|c|c|c|c|}
\hline \multirow{2}{*}{$\begin{array}{l}\text { Faixa Etária } \\
\text { (anos) }\end{array}$} & PTN & Vit A & Vit C & Vit E & $\mathrm{Fe}$ & $\mathrm{Zn}$ \\
\hline & (g/kg/dia) & ( $\mu \mathrm{g}$ RAE/dia) & (mg/dia) & (mg/dia) & (mg/dia) & (mg/dia) \\
\hline \multicolumn{7}{|l|}{$1-3$} \\
\hline EAR & 0,87 & 210,0 & 13,0 & 5,0 & 3,0 & 2,5 \\
\hline Média \pm DP & $3,90 \pm 1,78$ & $1271,7 \pm 299,8$ & $39,6 \pm 51,3$ & $3,5 \pm 0,4$ & $7,9 \pm 2,3$ & $4,0 \pm 0,5$ \\
\hline \multicolumn{7}{|l|}{$3-18$} \\
\hline EAR & 0,76 & 275,0 & 22,0 & 6,0 & 4,1 & 4,0 \\
\hline Média \pm DP & $2,65 \pm 0,97$ & $1242,3 \pm 1370,0$ & $35,8 \pm 48,6$ & $4,2 \pm 1,8$ & $9,3 \pm 2,7$ & $5,5 \pm 2,1$ \\
\hline \multicolumn{7}{|l|}{$8-13$} \\
\hline EAR & 0,76 & 445,0 & 39,0 & 9,0 & 5,9 & 7,0 \\
\hline Média \pm DP & $2,07 \pm 0,62$ & $2.018,2 \pm 2.883,1$ & $28,4 \pm 26,7$ & $5,5 \pm 2,8$ & $10,0 \pm 1,6$ & $5,0 \pm 1,4$ \\
\hline \multicolumn{7}{|l|}{$13-15$} \\
\hline EAR & 0,73 & 630,0 & 63,0 & 12,0 & 7,7 & 8,5 \\
\hline Média \pm DP & $1,82^{*}$ & $7.479,4 \pm 9.168,5$ & $29,5 \pm 15,3$ & $9,8 \pm 8,8$ & $10,5 \pm 1,5$ & $5,1 \pm 2,2$ \\
\hline
\end{tabular}

*O calculo da média não foi possível pois apenas um indivíduo deste grupo teve o peso corporal aferido.

* It was not possible to calculate the mean because only one individual in the group was weighed.

Tabela 4 - Necessidade média estimada (EAR) e média de consumo de nutrientes, de acordo com faixas etárias, em indivíduos menores de 16 anos do sexo feminino, residentes numa área de invasão em Maceió, Alagoas, 2005.

Table 4 - Estimated Average Requirement (EAR) and average consumption of nutrients according to age group, in female individuals under 16 years of age living in an area of invasion in Maceio, Alagoas, 2005.

\begin{tabular}{|c|c|c|c|c|c|c|}
\hline \multirow{2}{*}{$\begin{array}{l}\text { Faixa Etária } \\
\text { (anos) }\end{array}$} & PTN & Vit A & Vit C & Vit E & $\mathrm{Fe}$ & $\mathrm{Zn}$ \\
\hline & (g/kg/dia) & ( $\mu \mathrm{g}$ RAE/dia) & (mg/dia) & (mg/dia) & (mg/dia) & (mg/dia) \\
\hline \multicolumn{7}{|l|}{$1-13$} \\
\hline EAR & 0,87 & 210,0 & 13,0 & 5,0 & 3,0 & 2,5 \\
\hline Média \pm DP & $3,84 \pm 2,87$ & $1.512,2 \pm 502,6$ & $46,8 \pm 57,3$ & $3,5 \pm 0,4$ & $8,1 \pm 2,1$ & $4,0 \pm 0,7$ \\
\hline \multicolumn{7}{|l|}{$3-18$} \\
\hline EAR & 0,76 & 275,0 & 22,0 & 6,0 & 4,1 & 4,0 \\
\hline Média \pm DP & $2,54 \pm 0,84$ & $1.150,4 \pm 1.102,2$ & $48,8 \pm 73,9$ & $3,8 \pm 1,6$ & $9,4 \pm 2,6$ & $5,1 \pm 2,0$ \\
\hline \multicolumn{7}{|l|}{$8-13$} \\
\hline EAR & 0,76 & 420,0 & 39,0 & 9,0 & 5,9 & 7,0 \\
\hline Média \pm DP & $1,73 \pm 0,61$ & $2.153,0 \pm 3.642,0$ & $24,7 \pm 18,7$ & $5,6 \pm 3,4$ & $9,4 \pm 1,5$ & $5,1 \pm 1,4$ \\
\hline \multicolumn{7}{|l|}{$13-15$} \\
\hline EAR & 0,71 & 485,0 & 56,0 & 12,0 & 7,9 & 7,3 \\
\hline Média \pm DP & $1,67 \pm 1,26$ & $1.656,8 \pm 1.709,0$ & $21,6 \pm 16,5$ & $6,5 \pm 3,0$ & $10,8 \pm 2,1$ & $4,4 \pm 1,2$ \\
\hline
\end{tabular}


não hemínico. Esta vitamina atua também como co-fator em várias reações enzimáticas essenciais, sendo a mais importante a hidroxilação da prolina, indispensável para a formação do colágeno ${ }^{31,33}$.

Entre os minerais estudados, apenas o $\mathrm{Fe}$, apresentou média de consumo superior à recomendação nas faixas etárias avaliadas em ambos os sexos (Tabelas 3 e 4). Foram excluídos da avaliação de inadequação os indivíduos do sexo feminino maiores de 9 anos, devido à variação das necessidades diárias de Fe nesta população. Apesar da ingestão de Fe estar acima da média, a absorção deste mineral, na forma não hemínica, pode estar comprometida pelo baixo consumo de vitC. A Figura 2 apresenta a frequência de ingestão inadequada deste mineral nas duas faixas etárias avaliadas, cuja distribuição foi semelhante ( $p>0,05)$. O Fe é essencial para a expansão do volume sanguíneo e da massa muscular, exercendo funções metabólicas ou enzimáticas e de estoque para manter a homeostase. Quando sua ingestão não está adequada, os estoques no organismo são mobilizados para mater a produção de hemoglobina (Hb) e outros componentes que contêm $\mathrm{Fe}^{34}$. Convém ressaltar a elevada prevalência de anemia ferropriva no Brasil; segundo Batista-Filho, 40 a $50 \%$ das crianças menores de 5 anos são portadoras de anemia ${ }^{35}$.

Uma avaliação complementar sobre a ingestão alimentar de Fe poderia ser realizada calculando-se a biodisponilidade do Fe ingerido e separando-se o Fe total em Fe hemínico e não hemínico.

A média de ingestão de $\mathrm{Zn}$, foi inferior à recomendação nos indivíduos de ambos os sexos do grupo etário de 9 a 13 e 14 a 15 anos (Tabelas 3 e 4). O consumo inadequado de $\mathrm{Zn}$ foi superior a $20 \%$ em todos os grupos, sendo mais frequente entre os indivíduos de 1 a 3 anos $(\mathrm{p}<0,05)$ (Figura 2). O Zn é fundamental para o funcionamento adequado da imunidade e para o desenvolvimento cognitivo. A ingestão inadequada deste mineral pode levar a alterações bioquímicas e funcionais em condições fisiológicas que aumentam sua necessidade, como na fase de crescimento de crianças ou adolescentes. Uma das principais consequências da deficiência de Zn consiste na disfunção imune $^{36,37}$.

Em relação ao consumo dos micronutrientes avaliados segundo o método EAR

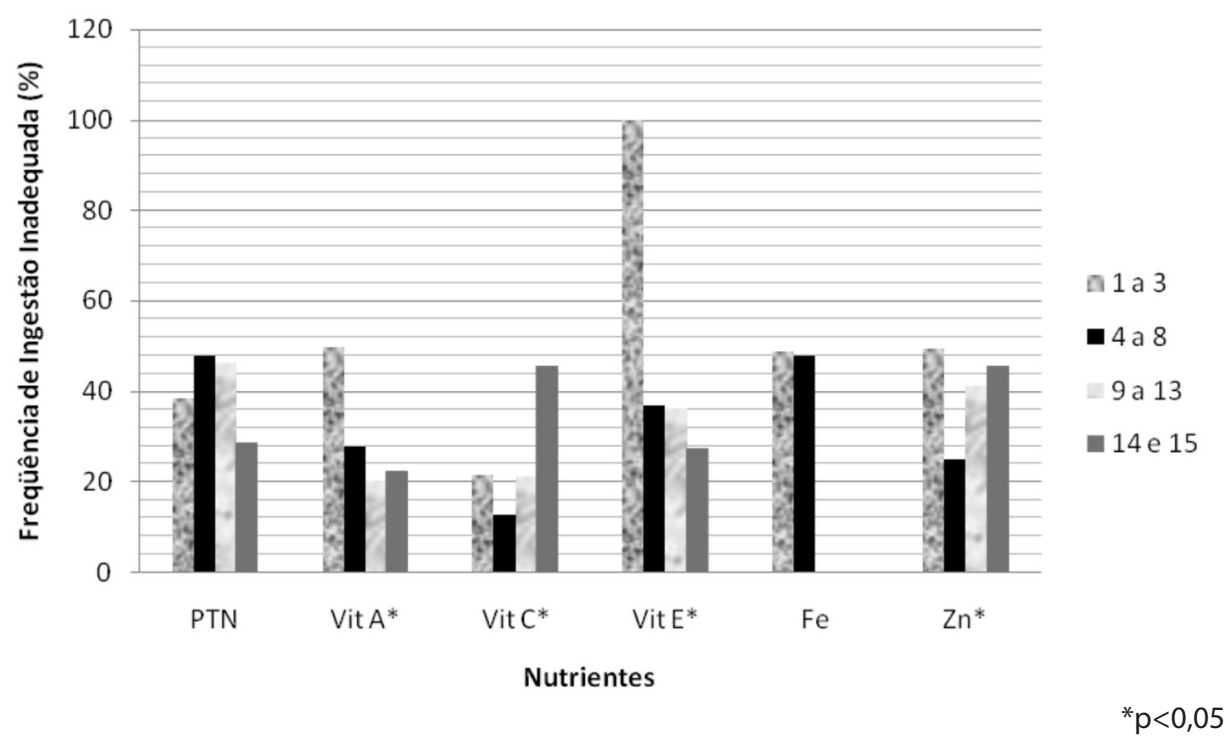

Figura 2 - Distribuição de ingestão inadequada de nutrientes, de acordo com as faixas etárias, em indivíduos menores de 16 anos, residentes numa área de invasão em Maceió, Alagoas, 2005. Figure 2 - Distribution of inadequate intake of nutrients according to age groups in individuals under 16 years of age living in an area of invasion in Maceio, Alagoas, 2005. 
Tabela 5 - Ingestão adequada (Al) e consumo médio de cálcio, de acordo com faixas etárias, em indivíduos menores de 16 anos, residentes numa área de invasão em Maceió, Alagoas, 2005.

Table 5 - Adequate Intake (Al) and average consumption of calcium according to age group, in individuals under 16 years of age living in an area of invasion in Maceio, Alagoas, 2005.

\begin{tabular}{lccc}
\hline Faixas etárias (anos) & $\mathrm{N}$ & $\mathrm{Al}(\mathrm{mg})$ & Média $\pm \mathrm{DP}(\mathrm{mg})$ \\
\hline $1-3$ & 56 & 500 & $774,05 \pm 238,47$ \\
$3 \dashv 8$ & 106 & 800 & $380,54 \pm 208,39$ \\
$8 \dashv 13$ & 99 & 1300 & $274,93 \pm 74,15$ \\
$13-15$ & 11 & 1300 & $284,20 \pm 68,47$ \\
\hline
\end{tabular}

como ponto de corte, a faixa etária de 1 a 3 anos foi a que apresentou maior deficiência. O padrão alimentar encontrado necessita de ajustes para prevenir carências nutricionais específicas que repercutem na saúde e no estado nutricional das crianças e adolescentes.

A Tabela 5 apresenta os valores de ingestão adequada (AI) e consumo médio de Ca nas faixas etárias estudadas. Apenas o grupo de 1 a 3 anos teve o consumo médio de Ca superior à AI. Nos demais grupos etários, o consumo médio foi aquém da recomendação e nenhuma conclusão pode ser estabelecida.

Durante os períodos de intenso desenvolvimento físico, a necessidade de Ca chega a triplicar, pois o osso maduro é constituído de $60 \%$ do peso dos minerais Ca e fósforo. Portanto, a ingestão inadequada deste mineral pode comprometer o crescimento esquelético. É consenso entre os especialistas que os indivíduos que não alcançam a maior densidade de massa óssea durante o pico de formação, apresentam maior risco de desenvolver osteoporose em idades mais avançadas $^{38,39}$.

A discussão destes resultados com a literatura não foi possível devido à ausência de trabalhos publicados utilizando dados corrigidos pela variabilidade intrapessoal.

\section{Conclusão}

Os dados demonstram que o padrão alimentar das crianças e adolescentes da área estudada necessita de ajustes, particularmente a faixa etária de 1 a 3 anos. A adequação da dieta pode prevenir deficiências nutricionais específicas que repercutem na saúde e no estado nutricional.

\section{Referências}

1. Marchioni DM, Slater B, Fisberg RM. Aplicação das dietary reference intakes na avaliação da ingestão de nutrientes para indivíduos. Rev Nutr 2004; 17: 207-16.

2. Tuma RC, Costa TH, Schmitz BA. Avaliação antropométrica e dietética de pré-escolares em três creches de Brasília, Distrito Federal. Rev Bras Saúde Matern Infant 2005; 5: 419-28.

3. Garcia GC, Gambardella AM, Frutuoso MF. Estado Nutricional e consumo alimentar de adolescentes de um centro de juventude da cidade de São Paulo. Rev Nutr 2003; 16: 41-50.

4. Fisberg M, Bandeira CRS, Bonilha EA, Halpern G, Hirschbruch. Hábitos alimentares na adolescência. Ped Moder 2000; 36: 724-34.
5. Willet W. Nature of variation in diet. In: Willet W. Nutritional Epidemiology. Oxford: Oxford University Press; 1998. p. 33-49.

6. Fisberg RM, Marchioni DML, Slater B. Aplicações das DRIs na avaliação da ingestão de nutrientes para grupos. In Sociedade Brasileira de Alimentação e Nutrição / Internacional Life Sciences Institute. Uso e aplicações das "Dietary Reference Intakes" DRIs. SBAN/ILSI ; 2001. p. 35-46.

7. Hoffmann K, Boeing H, Dufour A, Volatier JL, Telman J, Virtanen M, et al. Estimating the distribuition of usual dietary intake by short-term measurements. Euro J Clin Nutri 2002; 56: S53-S62. 
8. Cavalcante AA, Tinôco AL, Cotta RM, Ribeiro RC, Pereira CA, Franceschini SC. Consumo alimentar e estado nutricional de crianças atendidas em serviços públicos de saúde do município de Viçosa, Minas Gerais. Rev Nutr 2006; 19: 321-30.

9. Menezes RC, Osorio MM. Consumo energético-protéico e estado nutricional de crianças menores de cinco anos no estado de Pernambuco, Brasil. Rev Nutr 2007; 20: 337-47.

10. Fisberg RM, Martini LA, Slater B. Métodos de inquéritos alimentares. In Fisberg RM, Slater B, Marchioni DML, Martini LA, organizadores. Inquéritos alimentares: métodos e bases científicas. Barueri: Editora Manole; 2005. p. 2-24.

11. Zaboto CB, Vianna RPT, Gil MF. Registro fotográfico para inquéritos dietéticos: utensílios e porções. Campinas: Núcleo de Estudos e Pesquisas em Alimentação da Universidade Estadual de Campinas / Goiânia: Universidade Federal de Goiás; 1996.

12. Philippi ST, Szarfarc SC, Latterza AR. Virtual Nutri, Versão 1.0, for Windows [software]. São Paulo: Universidade de São Paulo, Faculdade de Saúde Pública, Departamento de Nutrição; 1996.

13. NEPA/UNICAMP (Núcleo de Estudos e Pesquisas em Alimentação da Universidade Estadual de Campinas). Tabela brasileira de composição de alimentos. Campinas: NEPA/UNICAMP; 2006.

14. IBGE (Instituto Brasileiro de Geografia e Estatística). Tabela de composição de alimentos. Rio de Janeiro: IBGE; 1977.

15. Pinheiro ABV, Lacerda EMA, Benzecry EH, Gomes MCS, Costa VM. Tabela para avaliação de consumo alimentar em medidas caseiras. São Paulo: Atheneu; 2004.

16. Martins MHS. Valor nutritivo de alimentos definidos por pesos médios, frações e medidas caseiras. Recife: Universidade Federal de Pernambuco; 1982.

17. IOM (Institute of Medicine). Dietary reference intakes: applications in dietary assessment. Washington: IOM; 2001.

18. Beaton GH. Ingestão dietética recomendada: indivíduos e populações. In: Shils ME, Olson JA, Shike M, Ross AC. Tratado de nutrição moderna na saúde e na doença. Barueri: Manole; 2003, p. 1829-51.

19. IOM (Institute of Medicine). Dietary reference intakes for energy, carbohydrate, fiber, fat, fatty acids, cholesterol, protein and amino acids (macronutrients). Washington: IOM; 2002.

20. SPSS (Statistical Package for the Social Sciences) for Windows [software]. Release 11.5. Chicago; 2002.

21. Schieri R, Coitinho DC, Pereira RA, Marins VMR, Moura AS. Variações temporais do estado nutricional e do consumo alimentar no Brasil. Physis Rev Saúde Col 1997; 7:31-50.
22. Levy-Costa RB, Sichieri R, Pontes NS, Monteiro CA. Disponibilidade domiciliar de alimentos no Brasil: distribuição e evolução (1974-2003). Rev Saúde Pública 2005; 39: 530-40.

23. Novaes JF, Franceschini SCC, Priore SE. Hábitos alimentares de crianças eutróficas e com sobrepeso em Viçosa, Minas Gerais, Brasil. Rev Nutr 2007; 20: 633-42.

24. Nunes MMA, Figueiroa JN, Alves JGB. Excesso de peso, atividade física e hábitos alimentares entre adolescentes de diferentes classes econômicas em Campina Grande (PB). Rev Assoc Med Bras 2007; 53: 130-4.

25. Marchini JS, Fausto MA, Rodrigues MMP, Oliveira JED, Vannucchi H. Necessidades e recomendações de proteínas: revisão atualização e sugestões. Cad Nutri 1993; 6: 1-21.

26. Amaral MFM, Morelli V, Pantoni RW, Rosseti-Ferreira UC. Alimentação de bebês e crianças pequenas em contextos coletivos: mediadores, interações e programas em educação infantil. Rev Bras Cresc Desenv Hum 1996; 6: 19-33.

27. Ambrósio C, Campos F, Faro Z. Carotenóides como alternativa contra a hipovitaminose A. Rev Nutr 2006; 19: 233-43.

28. Garcia-Casal MN, Layrisse M, Solano L, Barón A. Vitamin $A$ and $\beta$-carotene can improve nonheme iron absorption from rice, wheat and corn by humans. J Nutr 1998; 128: 646-50.

29. Osório MM. Fatores determinantes da anemia em crianças. J Pediatr 2002; 78: 269-78.

30. Ross AC. Vitamina A e retinóides. In: Shills ME, Olson JA, Shike M, Ross AC. Tratado de nutrição moderna na saúde e na doença. Barueri: Manole; 2003. p. 325-50.

31. Bricarello LP, Goulart RMM. O papel das vitaminas em lactentes e crianças. Rev Med Mod 1999; 35: 797-807.

32. Oliveira AF, Oliveira FLC, Juliano Y, Ancona-Lopez F. Evolução nutricional de crianças hospitalizadas e sob acompanhamento nutricional. Rev Nut 2005; 18: 341-48.

33. Amaya-Farfan J, Domene SM, Padovani R. DRI: Síntese comentada das novas propostas sobre recomendações nutricionais para antioxidantes. Rev Nutr 2001; 14: 71-8.

34. Urbano MR, Vitalle MS, Juliano Y, Amancio OM. Ferro, cobre e zinco em adolescentes no estirão pubertário. $J$ Pediatr 2002; 78: 327-34.

35. Batista-Filho M. O controle nas anemias no Brasil. Rev Bras Saúde Matern Infant 2004; 4: 121-3.

36. Mafra D, Cozzolino S. Importância do zinco na nutrição humana. Rev Nutr 2004; 1: 79-87.

37. Cunha,DF, Cunha SFC, Garcia-Junior A. Microminerais. In: Dutra-de-Oliveira JE, Marchini JS. Ciências Nuitricionais: aprendendo a aprender. São Paulo: Sarvier; 2008, p. 181-208. 
38. Heaney RP. Calcium in the prevention and treatment of osteoporosis. J Int Med 1992; 231: 169-180 In: Lerner BR, Lei DL, Chaves SP, Freire RD. O cálcio consumido por adolescentes de escolas públicas de Osasco, São Paulo. Rev Nutr 2000; 13: 57-63.
39. Schaafsma G. The scientific basis of recommended dietary allowances for calcium. J Intern Med 1992; 231: 187-94.

Recebido em: 22/04/09

Versão final reapresentada em: 27/08/09

Aprovado em: 30/09/09 